\title{
CUANDO SOMOS MÁS DE DOS
}

Rafael Sibils

Doctor en Medicina con posgrado en Psiquiatría, UDELAR

Miembro Habilitante de AUDEPP

Profesor adjunto del IUPA

Correo electrónico: rafaelsibils@gmail.com ORCID: 0000-0001-7434-1287 


\section{Resumen}

En este trabajo se describen las principales características, aspectos positivos y dificultades del tratamiento que integran psicoterapia y psicofarmacología. Se hace referencia a la perspectiva de pacientes y técnicos. Se enfatiza en las variables comunicacionales, el par transferencia-contratransferencia y el encuadre. Finalmente, se sugieren pautas para optimizar esta forma de trabajo.

Palabras clave: psicoterapia, psicofármaco, integración.

\section{When we are more than two}

\section{Abstract}

In this paper, we describe the main characteristics, positive aspects and difficulties of the treatment that constitute psychotherapy and psychopharmacology. Reference is made to the perspective of patients and technicians. Emphasis is placed on communicational variables, the transference-countertransference pair, and the frame. Finally, we suggest guidelines to optimize this way of working.

Keywords: psychotherapy, psychotropic drug, integration. 


\section{INTRODUCCIÓN}

Hay procedimientos psicoterapéuticos útiles prácticamente para toda la patología psíquica, y también recursos farmacológicos. Algunos problemas son abordables casi exclusivamente desde la psicoterapia y —en el otro extremo- determinadas patologías mejoran de modo decisivo con fármacos. Sin embargo, múltiples estudios coinciden en que la integración de psico y farmacoterapia es una estrategia de primera línea para mejorar los síntomas y la calidad de vida de los pacientes en la mayoría de los casos y que se utiliza cada vez con más frecuencia (Miklowitz et al., 2020; Lipovetzky y Agrest, 2006; Koenigsberg, 1994; De Jonghe et al., 2004; Kamenov et al., 2017; Lee y Hills, 2005; Torres-Torija, 2009; Cuijpers et al., 2009).

Ha habido cambios desde que los psiquiatras consideraban que el diálogo era solo parte del diagnóstico y no de la terapéutica y que los psicólogos creían que existía la posibilidad de algo así como un psiquismo sine materia. Parece claro que la psiquiatría debe ocuparse de la subjetividad y no limitarse a consejos y farmacología, y que la psicoterapia tiene que enfocar aspectos del paciente más allá de la pura subjetividad. Además, la integración de ambas perspectivas es más que un simple agregado de técnicas (Lipovetzky y Agrest, 2006). Hoy se sabe que los dos abordajes modifican la psiquis (emociones, pensamiento, acciones) y el cuerpo, particularmente el cerebro (Peedicayil, 2012; Linden, 2006; Kandel, 1998; Torres-Torija, 2009).

La literatura sobre tratamiento conjunto entre psiquiatras y terapeutas no es mucha, está fragmentada y proviene de diversos métodos de investigación. Sin embargo, Donatti et al. (2015) sostienen que 
trabajos de diversa índole refuerzan la idea de que el trabajo en colaboración puede ser explotado con múltiples ventajas siempre y cuando se integre en una estrategia coherente en cuanto a sus conceptos teóricos, al diseño de las intervenciones y al ensamblado ideológico del equipo tratante. (p. 30)

Más allá de ello, hay casos en los que es preferible solo medicación y otros en los que únicamente psicoterapia (Fernández-Álvarez et al., 2000). Este enfoque todavía genera dudas, discordancias, desencuentros y complicaciones; exige investigación, profundización y más práctica.

Muchos estudios refieren a cómo combinar tratamientos en situaciones específicas: patologías, momentos de los pacientes, problemas puntuales a partir de los cuales es difícil generalizar (Miklowitz et al., 2020; Koenigsberg, 1994; De Jonghe et al., 2004; Kamenov et al., 2017; Ray et al., 2020; Marazziti et al., 2020; Jindal y Thase, 2003). Frecuentemente concluyen en que es necesario continuar investigando, pero en este tema es difícil investigar cuantitativamente y generalizar conclusiones por el gran número de variables en juego (Gabbard y Kay, 2001; Driessen et al., 2020; Leichsenring et al., 2017; Fernández-Álvarez et al., 2000). De hecho, no se trata solo de utilizar fármacos y psicoterapia, sino que implica coordinar un espacio relacional entre al menos tres actores: paciente, terapeuta y psiquiatra; dicho espacio puede aportar a la mejoría del paciente, pero también comprometerla mediante errores diversos.

Hay varios términos para el tratamiento: combinado, en colaboración, dual, bifocal o bimodal, a su vez ligados a conceptos como equipo, multidisciplina, interdisciplina, pluridisciplina, transdisciplina e interconsulta (Azaretto y Ros, 2015), que refieren al tema, aunque eventualmente se pierden en disquisiciones un tanto abstrusas. Utilizaré aquí la expresión tratamiento integrado. Me parece clara, precisa y refiere a la combinación coherente de distintas modalidades terapéuticas. Ha sido utilizada 
en este tema sin mayores pretensiones conminativas o restrictivas, por lo que permite incursionar con libertad en él, tanto al redactar como al leer.

Las complejidades y los factores que atraviesan este campo van desde los más concretos, como la estructura sanitaria y las vías de comunicación entre técnicos, hasta los ideológicos, de poder, o las diferencias entre concepciones teóricas. No profundizaré en todos; mi objetivo es abordar los aspectos más habituales y prácticos que tienen lugar en tres grandes áreas, hecho determinante en esta forma de tratamiento:

consulta privada por profesionales independientes,

atención institucional pública,

atención institucional privada.

Cada área incluye múltiples variables: tiempos, locaciones, frecuencia, privacidad, costo para el paciente, remuneración de los técnicos, distancia geográfica, atención telefónica, virtual o presencial, etc., que generan diferentes grados de complejidad en los abordajes y facilitan o entorpecen la relación entre los profesionales.

Un objetivo de este artículo es interesar a los colegas en esta modalidad para optimizar los resultados terapéuticos (Ellison, 2005). Dadas las múltiples investigaciones y variables en juego, a la hora de plantear algunas ideas parece necesario agregar, a los estudios científicos puros y duros y a los criterios de expertos citados en las referencias bibliográficas, la experiencia propia y la de colegas de nuestro medio, una dosis de sentido común, y dejar al lector la tarea de diseñar la mejor estrategia en cada caso.

\section{TRATAMIENTO INTEGRADO}

A veces, un único profesional realiza las dos tareas, pero en general uno lleva adelante la psicoterapia y otro prescribe los fármacos. Si bien el enfoque que haré se apoya en conceptos psicoanalíticos, la mayor parte 
de la investigación no encuentra diferencias de resultado entre modelos psicoterapéuticos basados en evidencia (Torres-Torija, 2009).

La situación más frecuente y paradigmática es que un psiquiatra se encargue de la psicofarmacología y otro técnico, casi siempre un psicólogo, de la psicoterapia. Los dos son responsables, además de otros aspectos, de la atención del paciente: la relación con su familia y trabajo, las medidas médicas generales, el acompañamiento y consejo, la interacción con otros técnicos ligados a la salud mental o general, entre otros.

Ambas aproximaciones pueden estimular la adherencia al tratamiento. Los medicamentos facilitan la accesibilidad psicoterapéutica al fomentar funciones del yo valiosas para la psicoterapia y ejercen efectos positivos en expectativas y actitudes. La psicoterapia ayuda a dirimir preconceptos o problemas de los usuarios ante la psicofarmacología y a combatir el estigma que recae sobre los pacientes psiquiátricos.

También puede haber efectos negativos: la mejoría farmacológica de los síntomas podría disminuir la motivación para la psicoterapia y esta última podría ser sintomatológicamente disruptiva al remover conflictos. Estas hipotéticas situaciones y otras adyacentes tienen suficiente sustento como para considerar su posible vigencia en cada caso.

La integración fármacos-terapia parece ser importante en pacientes graves y en situaciones de riesgo, en tanto se suman abordajes, opiniones y controles. En oportunidades, surge frente a crisis; algunas implican riesgo vital (ideación o intento de suicidio, conductas autodestructivas graves, adicciones en carrera de consumo, etc.). Y, a veces, aparece frente a descompensaciones que requieren cambios en el abordaje que se está implementando.

Con frecuencia, uno de los profesionales indica el tratamiento integrado desde el inicio y deriva al paciente a un técnico de su confianza, lo que facilita la comunicación. Otras veces, el contacto se establece en el curso de la terapéutica porque el psiquiatra sugiere una psicoterapia o el psicólogo una consulta psiquiátrica. 
El intercambio entre psiquiatra, psicólogo, paciente y eventualmente su entorno conduce a mejor atención y capacidad de contención para el paciente y su familia, y de tal comunicación dependen en buena medida la eficacia y eficiencia de la terapéutica. Veamos algunos aspectos de cada uno de los participantes.

\section{El paciente}

Su edad, personalidad, psicopatología, postura ideológica frente a la farmacología y la psicoterapia, capacidades, vulnerabilidades y preferencias pueden orientar hacia cuál, cómo y cuándo será la mejor forma de incluir a un tercero en el tratamiento. La desconfianza, las fantasías, la vergüenza y la hetero y autoestigmatización conscientes e inconscientes, así como ciertas ideas y preferencias, aportan datos y señales para decidir acerca de la integración.

Con frecuencia, los técnicos manejan aspectos de la interacción con los pacientes mediante la lógica de las relaciones interpersonales cotidianas, sin observar en detalle elementos no conscientes, importantes a la hora de tomar decisiones.

Veamos algunas ideas que se presentan en los pacientes, que no agotan la variedad posible:

Uno de los dos profesionales sabe más y conduce el tratamiento o las variantes cercanas de esta formulación. (Por ejemplo, el psicólogo depende jerárquicamente del psiquiatra, quien determina finalmente qué es lo mejor. O bien, el psicólogo escucha y comprende más, y al psiquiatra le importa menos entender.) Es preciso que se le aclare bien la situación al paciente y, para ello, es importante que los técnicos tengan una estrategia común. Los campos de intervención de cada uno deben quedar definidos para todos y las eventuales diferencias de perspectiva 
deben tramitarse entre los profesionales, con objeto de presentar a la persona en tratamiento una versión lo más consistente posible.

Es lo mismo la consulta psicoanalítica (o de otra teoría psicoterapéutica) que la psiquiátrica. Es importante dejar claras las pertinencias y los objetivos de cada espacio. Hay datos que sirven para uno y son irrelevantes para el otro, y los técnicos tienen que hacérselo saber al paciente. Se pierde la absoluta privacidad de una relación entre dos. Es así y parece adecuado que el paciente, de común acuerdo con su terapeuta, pueda reservar información que el psiquiatra no tiene por qué compartir en tanto no es útil para el manejo farmacológico.

El uso de medicación implica mayor gravedad. No necesariamente es así; corresponde sobre todo al psiquiatra enfocar este tema, para dejar la terapia como campo más libre para las fantasías del paciente.

El paciente plantea que prefiere «manejarse por sí mismo» y no utilizar recursos farmacológicos, como si por hacerlo perdiera parte de su identidad o control sobre ella. Algunos pacientes - no tantos - señalan que no quieren terapia porque no desean depender de nadie y solicitan exclusivamente fármacos. Estos aspectos requieren acuerdo entre ambos profesionales ante el paciente, centrado en la idea de que tanto los fármacos como la terapia tienen como objetivo que él pueda ser la mejor versión de sí mismo y que tienden a su mayor libertad y capacidad de elección.

El paciente supone que los técnicos se comunican entre sí porque no trasmite bien los datos o hay aspectos a los que él no puede o debe acceder. Es necesario aclarar que no es así y que la comunicación se realiza para que los profesionales ajusten de la mejor manera su accionar.

Se desplazan de un profesional a otro ciertos aspectos o exigencias, consciente o inconscientemente. Parece natural que así sea y se trata —nuevamente- de delimitar los campos. 
El paciente supone que, luego del contacto entre los técnicos, toda la información fue intercambiada, ambos están al tanto en detalle de lo que está sucediendo y ya no le corresponde a él el esfuerzo y el involucramiento en la terapia. Esto genera que tiendan a depender de los técnicos, lo cual puede favorecer la reedición de la pareja parental u otras configuraciones familiares.

- El paciente desvaloriza o idealiza a uno de los técnicos y constituye - muchas veces con cierto acuerdo más o menos inconsciente del otro- una «alianza» inadecuada. A veces, compara lo que los profesionales dicen y se apoya en lo que uno plantea para sostener sus posiciones frente al otro, manipular la situación, atacar a uno de ellos o como manifestación de resistencias. La dilucidación de estos puntos corresponde fundamentalmente al tratamiento psicoterapéutico, al manejo de elementos transferenciales.

\section{El entorno del paciente}

Este elemento no siempre está presente en la comunicación, pero justamente los casos que requieren tratamiento integrado suelen ser complejos, por lo que se vuelve necesario que uno o ambos técnicos tengan contacto con la familia o incluso, eventualmente, con el medio social del paciente. Es conveniente que sea un solo profesional quien lo haga para liberar al otro de responsabilidad y evitar las dificultades en la comunicación que agregar actores conlleva.

Es posible que se necesite interactuar con instancias laborales, administrativas, legales, etc., o bien comunicarse con las instituciones que brindan la cobertura. Estos parecen más roles del psiquiatra que del psicoterapeuta, aunque obviamente habrá variantes. 


\section{Psicoterapeutas y psiquiatras}

Ambos profesionales seguramente tendrán diferencias en aspectos particulares (formación, concepciones personales, experiencia, orientación psicoterapéutica incluso dentro de la propia escuela) y contextuales (consulta privada o institucional, etc.). Estos puntos son relevantes a la hora de una derivación o interconsulta y es preciso tomarlos en cuenta para constituir un buen diálogo. También podrán estar atravesados por sesgos y fantasías; veamos algunos...

Los siguientes son algunos de los sesgos y las fantasías posibles en los psicoterapeutas (la mayoría psicólogos):

Suponer que los psiquiatras ven a los pacientes en profundidad y los tienen presentes del modo que sucede en una psicoterapia. En general, las condiciones laborales obligan a los psiquiatras a ver a muchas personas en tiempos breves y es imposible que todos estén accesibles en su memoria. Muchas veces, esto es percibido como desinterés o soberbia. Tener la expectativa de que los psiquiatras poseen siempre la capacidad de precisar el diagnóstico o determinar con exactitud el riesgo $\mathrm{u}$ otros elementos, lo que puede no ser así: muchas veces son necesarias varias entrevistas para efectuar una serie diagnóstica adecuada y, para ello, los aportes del psicólogo son de gran valor, sobre todo en cuadros ambiguos en los que se requiere evaluación continua.

Compartir en diversa medida alguna de las falsas creencias o prejuicios acerca de la psiquiatría de muchas personas: los medicamentos producen adicción, la electroconvulsoterapia destruye neuronas y debe ser evitada, la internación es negativa. No necesariamente son conscientes de hasta qué punto estos sesgos influyen y es relativamente frecuente evidenciarlos.

Plantear que el sufrimiento sintomático debe ser parte del tratamiento y que los efectos farmacológicos afectarían la capacidad de resolver 
conflictos. Se conceptualiza así la farmacología solo como tratamiento coadyuvante, cuando en realidad ambas vertientes confluyen y se potencian para mejorar la patología de base.

Utilizar en ocasiones un lenguaje que proviene de concepciones psicoterapéuticas, no contemplado en la formación de todos los psiquiatras.

- Preocuparse por que, si disminuye la sintomatología, el paciente podría dejar la terapia y habría un paciente menos en su consulta. Es una variante de «el psiquiatra se va a quedar con el paciente».

A veces, le «explican» al psiquiatra qué tiene el paciente y lo que debería hacer, y la frustración y la rispidez pueden emerger de tal actitud. A continuación, se listan algunos de los sesgos y las fantasías posibles en los psiquiatras:

Muchas veces, desvalorizan la tarea del psicoterapeuta, en particular si es psicólogo. No necesariamente son conscientes de tal extremo, pero suponen que el verdadero tratamiento es el que ellos hacen y desconocen la psicoterapia.

Pueden tener el prejuicio de que el psicólogo no sabe de diagnóstico o no le interesa. Desacreditan su formación y enfoque desde una perspectiva médica omnipotente (Lipovetzky y Agrest, 2006).

A veces, le «explican» al psicólogo qué tiene el paciente desde una nosografía tomada como la única posible o tienden a decirle al terapeuta lo que debería hacer; la frustración y la rispidez son el corolario. Eventualmente —sobre todo si tienen formación en psicoterapiasienten que, si no aplican una escucha profunda y se mantienen en un nivel clínico, no demuestran conocimiento. Se puede tener una perspectiva —es imposible no hacerlo— que incluya aspectos psicodinámicos y vaya más allá de la psiquiatría clásica, pero hay que ser cuidadoso a la hora de efectuar intervenciones para no interferir en la terapia. La tentación se puede contener hasta comunicarse con el terapeuta. 


\section{Múltiples y complejas transferencias y contratransferencias}

La variedad de ejes transferenciales-contratransferenciales que atraviesan el campo entre los tres actores (eventualmente más si agregamos familia y allegados del paciente) es inabarcable. Se presentan en toda comunicación humana y pueden incidir para bien o para mal en el tratamiento, lo que hace necesario prever y reconocer a tiempo los que sea posible. Lee y Hill (2005) observan que puede generarse splitting en el paciente, conflictos entre los técnicos y terminación prematura del tratamiento por el paciente o uno de los profesionales.

Las vivencias que los técnicos tienen cuando su trabajo es observado por un par remiten a sus historias personales, se expresan de modo consciente o no y pueden ser fuente de interferencias, desconfianza, competencia, idealizaciones y otras posibilidades que afectan el tratamiento.

En tanto los psiquiatras están habilitados para realizar fármaco y psicoterapia, los terapeutas se limitan a esta última, lo que puede ser un área de rivalidad no del todo consciente. Algunos terapeutas pueden vivenciar la interconsulta como admisión de un supuesto fracaso e importa detectar una eventual transferencia negativa hacia el psiquiatra. Este último también puede convertirse en «objeto bueno» para el paciente en la transferencia dividida, especialmente cuando se da una mejora sintomática con medicamentos. Tal idealización puede ser utilizada por el paciente para resistir la exploración de la transferencia dolorosa; también podría refugiarse en un rol pasivo mientras espera el alivio farmacológico, lo que afecta su participación en la psicoterapia.

Algunos pacientes pueden percibir la consulta psiquiátrica inconscientemente como «abandono» por el terapeuta, como encuentro de dos figuras parentales o tener falsas expectativas acerca de la omnipotencia fantaseada del par de profesionales. 
El psiquiatra puede quedar en medio de una reacción transferencial negativa del paciente hacia el terapeuta, con las implicancias que esto conlleva desde el punto de vista del manejo. En tales casos, el paciente puede intentar convertir la consulta psiquiátrica en una sesión de terapia, quizás para evitar la ansiedad del enfrentamiento con el objeto del conflicto.

Los pacientes pueden imaginar a los técnicos como rivales, perseguidores en connivencia, y un amplio etcétera de posibilidades conscientes e inconscientes que habrá que investigar y tener en cuenta en cada caso. Asimismo, los efectos adversos de los medicamentos pueden convertirse en foco de la terapia para evitar otros temas, con los elementos transferenciales subsiguientes. Corresponde a ambos técnicos abordar este asunto cada uno desde su perspectiva.

En fin, las variantes son inabarcables en cuanto a posibilidades, por lo cual los técnicos tendrán que estar atentos a evidenciarlas.

\section{Encuadre en los tratamientos combinados}

En términos generales, el encuadre surge de la relación profesional e implica ciertas constantes que permiten el proceso psicoterapéutico. Su propósito es disminuir las interferencias y aportar estabilidad, privacidad y confianza, para dar lugar a lo que Winnicott (1971) denominó ambiente facilitador (en la crianza; en la terapia agrego yo), que permite contención, sostén y regulación.

El encuadre se funda en los conceptos de cada teoría, las características y la personalidad del terapeuta (disposición, actitud, experiencia, ética) y el compromiso del paciente. Bleger (1967) refiere que Winnicott define el setting como «la suma de todos los detalles de la técnica» (p. 103) y plantea que el encuadre es un no-proceso que enmarca el proceso terapéutico. Bleger (1967) considera el rol del analista, el conjunto de factores espaciotemporales y parte de la técnica (horarios, honorarios, interrupciones 
regladas, etc.), y propone el psicoanálisis del encuadre psicoanalítico como eje relevante.

En los tratamientos integrados todas estas alternativas se multiplican dadas la complejidad y la cantidad de relaciones reales y fantaseadas. Por tanto, asirse a reglas fijas y estrictas no parece operativo ni posible; la mejor opción parece ser un encuadre abierto y flexible, que a la vez exige responsabilidad para mantener una estructura que habilite el tratamiento. Esto recae en gran medida sobre el terapeuta, quien debe considerar las pautas de comunicación con el psiquiatra antes planteadas y acordar con él los términos de relación. Es un encuadre que multiplica y abre en gran medida las variables, y que está conformado sobre todo por la actitud, profesionalidad y receptividad de los técnicos; no tanto por los aspectos formales de la relación, aunque estos también tendrán cierta importancia.

\section{ALGUNAS PAUTAS}

Tomar en cuenta los ya mencionados sesgos de pacientes, psiquiatras y terapeutas, los aspectos transferenciales-contratransferenciales y el encuadre facilitará el tratamiento integrado. Además, entiendo posible señalar ciertas orientaciones, así como advertir acerca de determinadas dificultades — más allá de la complejidad y lo variado de los contextos-, para que tal tratamiento resulte más eficaz y eficiente.

En términos generales, el intercambio psiquiatra-psicólogo es acerca de aspectos diagnósticos y terapéuticos, y disponer de una pauta sencilla y flexible facilita la comunicación. Esta pauta podría acercarse a la siguiente:

Motivo de consulta.

Diagnóstico clínico (nosográfico, positivo, psiquiátrico).

Rasgos de personalidad. 
Diagnóstico psicopatológico, centrado en los mecanismos más significativos.

Enfermedades físicas relevantes.

Antecedentes personales y familiares.

Entorno familiar y psicosocial, de más o menos importancia en cada caso.

Posibles riesgos y actings.

Evaluación de la actividad global del paciente.

Manipulaciones que el paciente pueda intentar.

Elaboración o perspectiva conjunta de un proyecto terapéutico que incluya psicoterapia y farmacología (eventualmente paraclínica, farmacología general, electroconvulsoterapia, dieta, ejercicio, organización de las actividades, interconsulta con otros técnicos) y también instancias de apoyo familiar, social, institucional, grupal, de onG, laboral, etcétera.

Esbozo de un pronóstico.

Pautas para continuar el tratamiento integrado (formas de comunicación, selección de qué aspectos tratará cada uno con el paciente, entre otras).

Frecuentemente, son relevantes la intensidad y la urgencia del problema, el motivo de consulta, las capacidades psicológicas del paciente, su modalidad pasiva o activa, su ideología respecto a los recursos terapéuticos y la relación costo-beneficio (Fernández-Álvarez et al., 2000).

Si los técnicos se conocen o tienen referencias sobre el otro, se facilita la situación desde el inicio, mas no siempre es así y hay que ir estructurando las formas y la comunicación sobre la marcha. Si es la primera vez que trabajan en conjunto, les sería útil informarse acerca de la experiencia, orientación terapéutica, técnicas y especialización de cada uno y tomar en cuenta la estructura asistencial en la que se realizará el tratamiento. Es posible acordar —explícitamente o no- un lenguaje común 
que evite conflictos entre aspectos teóricos y referencias de las distintas disciplinas.

El uso de la psicoterapia se ha extendido en nuestro país desde la implantación del plan de prestaciones asistenciales en 2011, lo que facilita el acceso a los registros si ambos profesionales brindan atención en la misma institución, a la que podrán solicitar condiciones que mejoren la comunicación. El uso de una única historia clínica es una posibilidad cada vez más accesible, que permite ver lo que el otro técnico está privilegiando en la evolución del caso.

Ambos técnicos deberán reflexionar e intercambiar sobre las fantasías, manejos y aspectos transferenciales-contratransferenciales que surjan desde el paciente, pero también acerca de los que se dan entre ellos mismos. En la comunicación directa, el mail parece una buena elección en tanto da más tiempo a pensar, consultar registros y expresar ideas con claridad. Es positivo manifestar concordancias y discrepancias, aunque no involucrarse en discusiones acerca del marco teórico referencial de cada profesional. Naturalmente, no hay que evidenciar desacuerdos o críticas frente al paciente o familiares antes de dirimirlas con el otro técnico, en tanto esa actitud puede comprometer las bases del tratamiento.

Un problema puede ser que uno de los técnicos tenga información acerca de determinado punto y el otro no; habrá que decidir qué información se comparte y cuál debe reservarse (especialmente por el terapeuta). Asimismo, importa aclararle al paciente que, si está en terapia, lo relevante en la consulta psiquiátrica es la sintomatología.

Las anteriores propuestas y reflexiones exigen flexibilidad y creatividad, y creo que aportan para que los profesionales tratantes puedan referirse a la persona en tratamiento como nuestro paciente. 


\section{REFERENCIAS BIBLIOGRÁFICAS}

Azaretto, C. y Ros, C. B. (2015). Las relaciones del psicoanálisis y otros campos de saber en términos de multidisciplina-interdisciplina-transdisciplina. Memorias del VII Congreso Internacional de Investigación y Práctica Profesional en Psicología. XXII Jornadas de Investigación, XI Encuentro de Investigadores en Psicología del MERCOSUR. Facultad de Psicología, Universidad de Buenos Aires.

Bleger, J. (1967). Psicoanálisis del encuadre psicoanalítico. Revista de Psicoanálisis, 24(2), 241-258.

Cuijpers, P., Van Straten, A., Warmerdam, L. y Andersson, G. (2009). Psychotherapy versus the combination of psychotherapy and pharmacotherapy in the treatment of depression: a meta analysis. Depression and anxiety, 26(3), 279-288. https://onlinelibrary.wiley. com/doi/epdf/10.1002/da.20519

De Jonghe, F., Hendricksen, M., Van Aalst, G., Kool, S., Peen, V., Van, R., Van Den Eijnden, E. y DekKer, J. (2004). Psychotherapy alone and combined with pharmacotherapy in the treatment of depression. The British Journal of Psychiatry, 185(1), 37-45.

Donatti, S., Garay, C. J. y Etchevers, M. J. (2015). La comunicación entre psicólogos clínicos y psiquiatras en el tratamiento combinado (psicoterapia y farmacoterapia) en salud mental. Anuario de investigaciones, 22, 29-38.

Driessen, E., Dekrer, J. J., Peen, J., Van, H. L., Maina, G., Rosso, G., Rigardetto, S., Cuniberti, F., Vitriol, V. G., Florenzano, R. U., Andreoli, A., Burnand, Y., López-Rodríguez, J., Villamil-Salcedo, V., Twisk, J. W. R. y CUIJPERs, P. (2020). The efficacy of adding short-term psychodynamic psychotherapy to antidepressants in the treatment of depression: A systematic review and meta-analysis of individual 
participant data. Clinical Psychology Review, 80(101886). https://doi. org/10.1016/j.cpr.2020.101886

ELLISON, J. M. (2005). Teaching collaboration between pharmacotherapist and psychotherapist. Academic Psychiatry, 29(2), 195-202.

Fernández-Álvarez, H., Hirsch, H., Lagomarsino, A. y Pagés, S. (2000).

Psicoterapia y psicofármacos ¿Cómo y cuándo combinarlos? Vertex, Revista Argentina de Psiquiatría, 11(39), 5-12.

GABBARD, G. O. y KAY, J. (2001). The fate of integrated treatment: whatever happened to the biopsychosocial psychiatrist? American Journal of Psychiatry, 158(12), 1956-1963.

Jindal, R. D., y Thase, M. E. (2003). Integration of care: integrating psychotherapy and pharmacotherapy to improve outcomes among patients with mood disorders. Psychiatric Services, 54(11), 1484-1490. Kamenov, K., Twomey, C., Cabello, M., Prina, A. M. y Ayuso-Mateos, J. L. (2017). The efficacy of psychotherapy, pharmacotherapy and their combination on functioning and quality of life in depression: a meta-analysis. Psychological Medicine, 47(3), 414-425.

KANDEL, E. R. (1998). A new intellectual framework for psychiatry. American Journal of Psychiatry, 155(4), 457-469.

Koenigsberg, H. W. (1994). The combination of psychotherapy and pharmacotherapy in the treatment of borderline patients. The Journal of Psychotherapy Practice and Research, 3(2), 93-107.

LeE, T. S. W. y Hills, O. F. (2005). Psychodynamic perspectives of collaborative treatment. Journal of Psychiatric Practice, 11(2), 97-101.

Leichsenring, F., Abbass, A., Hilsenroth, M. J., Leweke, F., Luyten, P., Keefe, J. R., Midgley, N., Rabung, S., Salzer, S. y Steinert, C. (2017). Biases in research: risk factors for non-replicability in psychotherapy and pharmacotherapy research. Psychological Medicine, 47(6), 1000-1011. 
LiNDEN, D. E. J. (2006). How psychotherapy changes the brain-the contribution of functional neuroimaging. Molecular Psychiatry, 11(6), 528-538.

Lipovetzky, G. y Agrest, M. (2006). Aspectos clínicos de los tratamientos en colaboración entre psiquiatras y psicoterapeutas. Vertex, Revista Argentina de Psiquiatría, 17(68), 263-269.

Marazziti, D., Pozza, A., Avella, M. T. y Mucci, F. (2020). What is the impact of pharmacotherapy on psychotherapy for obsessive-compulsive disorder? Expert Opinion on Pharmacotherapy, 21(14), 16511654. www.tandfonline.com/doi/epub/10.1080/14656566.2020.1 775814?needAccess $=$ true

Miklowitz, D. J., Efthimiou, O., Furukawa, T. A., Scott, J., Mclaren, R., GedDES, J. R. y CipRIANI, A. (2020). Adjunctive psychotherapy for bipolar disorder: A systematic review and component network meta-analysis. JAMA Psychiatry, 78(2), 141-150. doi: 10.1001/ jamapsychiatry.2020.2993

PeedicayiL, J. (2012). Role of epigenetics in pharmacotherapy, psychotherapy and nutritional management of mental disorders. Journal of Clinical Pharmacy and Therapeutics, 37(5), 499-501.

Ray, L. A., Meredith, L. R., Kiluk, B. D., Walthers, J., Carroll, K. M. y MaGiLl, M. (2020). Combined pharmacotherapy and cognitive behavioral therapy for adults with alcohol or substance use disorders: a systematic review and meta-analysis. JAMA Network Open, 3(6). https://jamanetwork.com/journals/jamanetworkopen/fullarticle /2767358

Torres-ToriJA, J. (2009). Psicoterapia y psiquiatría: una relación paradójica. Salud mental, 32(3), 185-187. www.scielo.org.mx/pdf/sm/ v32n3/v32n3a1.pdf

WinNicotT, D. W. (1971). Playing and reality. Tavistock. 
